\title{
Crisis management during anaesthesia: vascular access problems
}

\section{R J Singleton, S B Kinnear, M Currie, S C Helps}

Qual Saf Health Care 2005;14:e20 (http://www.qshc.com/cgi/content/full/14/3/e20). doi: 10.1136/qshc.2002.004507

See end of article for authors' affiliations

.....................

Correspondence to: Professor W B Runciman, President, Australian Patient Safety Foundation GPO Box 400, Adelaide, South Australia, 5001 Australia; research@apsf. net.au

Accepted 12 January 2005
Background: In confronting an evolving crisis, the anaesthetist should consider the vascular catheter as a potential cause, abandoning assumptions that the device has been satisfactorily placed and is functioning correctly.

Objectives: To examine the role of a previously described core algorithm "COVER ABCD-A SWIFT $\mathrm{CHECK}^{\prime \prime}$, supplemented by a specific sub-algorithm for vascular access problems, in the management of crises occurring in association with anaesthesia.

Methods: The potential performance of a structured approach was evaluated for each of the relevant incidents among the first 4000 reported to the Australian Incident Monitoring Study (AIMS).

Results: There were 128 incidents involving problems related to vascular access. The structured approach begins distally, checking the infusion device or fluid (12 incidents), moving proximally by way of the fluid giving line (10), the line deadspace (8), then the catheter/skin interface (65), and on to the peripheral vascular tree (3) and central venous space (23), and finally, the interface of the vascular access system and the attending staff (7). The approach was able to accommodate all the vascular access problems among the first 4000 incidents reported to AIMS.

Conclusion: The approach has potential as an easily remembered and applied clinical tool to lead to early resolution of vascular access problems occurring during anaesthesia.
$\mathrm{T}$ he cause of a perioperative crisis is not always readily apparent to the anaesthetist. A problem may arise directly from the immediate actions of the attending theatre staff, or it may result from a later complication of a previous intervention. In this latter event, because the crisis may have arisen from actions taken before the patient reached the operating room, the potential exists for the cause of the problem to be beyond the anaesthetist's immediate frame of reference, further hindering the diagnostic process.

The great majority of patients presenting for anaesthesia and surgery require the establishment and ongoing use of some type of vascular access. Patients may present to anaesthetists with vascular access already apparently established, or the anaesthetist may be required to establish the access. In confronting an evolving crisis situation, the anaesthetist should always consider the ubiquitous vascular catheter as a potential cause for the emergency, abandoning any assumption that the device has been satisfactorily placed and is functioning correctly.

In 1993, a "core" crisis management algorithm, represented by the mnemonic COVER ABCD-A SWIFT CHECK (the $\mathrm{AB}$ precedes COVER for the non-intubated patient), was proposed as the basis for a systematic approach to any crisis during anaesthesia where it is not immediately obvious what should be done, or where actions taken have failed to remedy the situation. ${ }^{1}$ This was validated against the first 2000 incidents reported to the Australian Incident Monitoring Study (AIMS). AIMS is an ongoing study which involves the voluntary, anonymous reporting of any unintended incident which reduced, or could have reduced, the safety margin for a patient. $^{2}$

It was concluded that if this algorithm had been correctly applied, a functional diagnosis would have been reached in $40-60$ seconds in $99 \%$ of applicable incidents, and the learned sequence of actions recommended by the COVER portion would have led to appropriate steps being taken to handle the $60 \%$ of problems relevant to this portion of the algorithm. ${ }^{1}$ However, this study also showed that the $40 \%$ of problems represented by the remainder of the algorithm, ABCD-A SWIFT CHECK, were not always promptly diagnosed or appropriately managed. ${ }^{1-3}$ It was decided that it would be useful, for these remaining problems, to develop a set of sub-algorithms in an easy to use crisis management manual. ${ }^{4}$ This study reports on the place of the COVER ABCD-A SWIFT CHECK algorithm in the diagnosis and initial management of vascular access problems and provides an outline of a specific crisis management sub-algorithm for these problems during anaesthesia, based on a geographical approach to the patient, and provides an indication of the potential value of using this structured approach.

\section{METHODS}

Of the first 4000 incidents reported to AIMS, those that made reference to incidents involving vascular access were extracted and analysed for relevance, presenting features, type of surgery, cause, management, and outcome. The COVER ABCD-A SWIFT CHECK algorithm, described elsewhere in this set of articles, ${ }^{4}$ was applied to each relevant report to determine the stages at which the problem might have been diagnosed and to confirm that activating the COVER portion would have led to appropriate initial steps being taken. As vascular access problems are not dealt with by this algorithm, a specific sub-algorithm was developed for these problems (see figure), and its putative effectiveness was tested against the reports. The potential value of this structured approach (that is, the application of COVER ABCD-A SWIFT CHECK to the diagnosis and initial management of this problem, and the application of the relevant subalgorithm for vascular access problems) was assessed in the light of AIMS reports by comparing its potential effectiveness for each incident with that of the actual management, as recorded in each report.

\section{RESULTS}

Of the first 4000 reports reported to AIMS, 128 described incidents involving vascular access. Applying the mnemonic 


\section{VASCULAR ACCESS PROBLEMS A Sub-Algorithm}

Approach the diagnosis and management of these problems from a "geographical" perspective:

1. Infusion device/fluid

2. Fluid line/equipment

3. Line deadspace

4. Catheter/skin interface

5. Peripheral vascular tree

6. Central vascular space

7. Staff and environment

\section{EMERGENCY CHECK}

Review all ampoules/syringes/bags/burettes/diluents/cassettes Review all drug labels

Systematically review all vascular access equipment

Work from fluid source via the cannula/skin interface to the tip Check vehicles/dilations/rates/routes are correct

Correlate doses with effects

If you have any doubts about the contents of a syringe or infusion discard and prepare a new solution.

\section{ALWAYS CONSIDER}

Wrong patient

Wrong drug (3)*

Ampoule or syringe swap

Known allergy

Right drug (3)

Wrong dose/rate

Wrong diluent/dilution

Wrong time

Wrong route/site

Failure of intended delivery

Malpositioned cannula tip (1)

Bag/syringe/burette empty

Cannula disconnected/blocked/kinked (2)

Line disconnected/blocked/kinked

Back flow (up a "Y piece")

Unintended delivery (3)

Drug flushed from dead space of IV line or cannula (4)

Drip/pump delivering drug inadvertently (5)

"Flushing" syringe containing drug

Someone else giving the drug

Check for any unrecognised cannulae in situ (5)

\section{BEWARE}

Any syringe containing relaxant

Haematoma

Inadvertent intra-arterial placement

Pneumothorax/haemothorax/hydrothorax

Nerve damage

Dysrhythmias (cannula tip in heart)

Cannula dead space

This comprises a page of the Crisis Management Manual5.

* Numbers in brackets refer to Notes below.

NOTES:

(1) $51 \%$ of the 128 cases involved a fault at the catheter/skin interface. In $15 \%$ of these the cannula was inserted by someone other than the anaesthesiologist. 18\% involved central venous lines.

(2) Incorrect manufacture or assembly of arterial/central/peripheral lines $-8 \%$. Disconnection resulting in unrecognised blood loss $-6 \%$.

(3) Errors included:

Connection of wrong infusion pump/fluid - $3 \%$

Over/underdosage fluid/drugs - $2 \%$

Drug precipitation in the line dead space $-2 \%$

Anaphylaxis to infusions - $2 \%$.

(4) Suxamethonium remaining in the dead space caused late paralysis upon subsequent line flushing in 6 case reports.

(5) Unvisualised cannulae beneath drapes may be the source of disconnections, or be the route of an unknown delivery of drugs/fluids.

Corresponding notes to be shown on the reverse side of the page of the Crisis Management Manual5.

Figure 1 Vascular access problems: a sub-algorithm.

of the crisis management algorithm, "COVER ABCD", to these incidents, all belonged to the category covered by " $\mathrm{R}$ ""review all (vascular) equipment". As this is a very broad category encompassing many problems, these incidents were further analysed using a sub-algorithm based on a "geographical" approach to the patient (fig 1). Using this subalgorithm the anaesthetist proceeds with a systematic review of vascular access equipment beginning distally at the infusion device or fluid, and moving proximally by the way of the fluid giving line, the line deadspace, then the catheter/ skin interface, and onto the peripheral and central vascular tree. The incidents reported to AIMS are presented in groups below, according to categories based upon the use of the described sub-algorithm.

\section{Infusion device or fluid ( 12 cases)}

In four cases, a problem arose because the wrong infusion pump or solution had been inadvertently connected to the patient. In a further three reports, incidents occurred because an infusion pump was giving an unrecognised overdose. In three cases, anaphylaxis to the infusion fluid was finally recognised as the cause of the problem. In two patients, a dose of a drug from the deadspace of an infusion pump was eventually recognised as the causative event of an incident.

\section{Fluid line and equipment problems (10 cases)}

Five arterial lines and three central venous lines were faulty in either their manufacture or assembly at the bedside, thus creating problems in their insertion or use for the measurement of vascular pressures. Similar problems occurred with two peripheral lines: in one case, poor preparation almost allowed a large air embolus from an unprimed blood warming coil, and in another case a leak at the hub of an intravenous cannula put the patient at increased risk.

\section{Line deadspace (eight cases)}

In six patients, drug remaining in the deadspace of the intravenous cannula caused late muscle paralysis upon 
subsequent injection of fluid through the vascular port Succinylcholine was the drug in all cases.

There were two incident reports describing anaesthetists inadvertently mixing two drug solutions by their sequential injection into the deadspace of the intravenous line, resulting in precipitation in the line deadspace, causing occlusion at an inopportune time. On both occasions, the drugs were thiopentone and vecuronium.

\section{Catheter/skin interface (65 cases)}

There were 21 incidents of intravenous catheters assumed to be intravascular which proved to be located extravascularly upon attempted use. In 10 of these, the anaesthetist did not insert the catheter himself. There were 19 cases of assumed intravenous catheter placement actually later realised to be intra-arterial in position. Twelve incident reports described extravasation or dislodgment of a catheter upon unplanned movement of the patient. These catheters were not adequately secured. There were eight cases of a vascular catheter becoming unknowingly disconnected causing unrecognised blood loss; these incidents occurred when the catheter was hidden from the anaesthetist by the surgical drapes. Two incident reports described fragments of catheter remaining in the patient upon device removal. There was a single incident of an intravenous catheter being placed inappropriately on an arm with an arterio-venous fistula, another of the catheter insertion being delegated to an inappropriately trained individual, and one incident of continued inadvertent intraarterial injection of drug despite considerable pain on injection.

\section{Peripheral vascular tree (three cases)}

There was one incident report of an arterial line causing thrombosis, and one report of a radial arterial line producing hand ischaemia. Another incident involved an intravenous catheter in the antecubital fossa damaging the brachial artery.

\section{Central venous space ( 23 cases)}

There were five reports arising from attempted central venous catheterisation via an internal jugular vein. Three incidents described puncture of the cuff of the endotracheal tube by the advancing needle of an attempted jugular cannulation. Two other incidents described the development of a large neck haematoma.

Central venous cannulation by the subclavian route produced five reports of pneumothorax, four reports of a pleural effusion or hydrothorax, and one case of haemothorax. There was one central venous and one central arterial thrombosis reported, and one description of cardiac tamponade after subclavian needle passage. One incident outlined a failed subclavian vein catheterisation and the reporter attributed the lack of success to unrecognised hypovolaemia. Four incidents described the acute onset of dysrhythmia with the insertion of a central venous catheter.

\section{Staff and situation problems (seven cases)}

Three incidents described the loss of function of the vascular catheter produced by the surgeon leaning on the equipment site. Three needlestick injuries were reported, and in each instance the injury was suffered not by the clinician inserting the needle, but by an assistant. One incident arose because of the failure of the staff at the transfer of the patient to communicate the presence of an intra-arterial catheter.

\section{DISCUSSION}

This descriptive paper illustrates that the problems associated with vascular access are many and varied in nature, and may not necessarily arise solely from the actions of the

\section{Key messages}

- Of the first 4000 anaesthesia incident reports to AIMS there were 128 incidents involving problems related to vascular access.

- All of these incidents belonged to the " $\mathrm{R}^{\prime \prime}$ - "review all (vascular) equipment" step in the COVER ABCD algorithm. The further application of the specific vascular access sub-algorithm is based upon a "geographical" approach with the anaesthetist's checking beginning distally and moving centrally stepwise.

- The resultant categories were: infusion device or fluid (12 cases); fluid line and equipment problems (10 cases); line deadspace (8 cases); catheter/skin interface (65 cases); peripheral vascular tree (3 cases); central venous space (23 cases); staff and situation problems (7 cases).

- The resolution of some vascular access problems may be facilitated by the additional application of the subalgorithm for problems related to drug administration, described elsewhere in the series of papers.

- Twelve reports of extravasation or dislodgement of a catheter were the result of unplanned patient movement.

- Subclavian venous cannulation produced five reports of pneumothorax, four reports of pleural effusion or hydrothorax, and one case of haemothorax.

- Three incidents of loss of catheter function resulted from the surgeon leaning on the site.

- The correct application of the core algorithm, followed by the specific sub-algorithm employing the geographical approach, was considered to accommodate all the vascular access problems among the first 4000 AIMS incidents.

anaesthetist. In any crisis or unsolved patient dilemma, the clinician should always consider the vascular access system as a potential cause for the problem. In the first instance, this will be accomplished by applying the crisis management mnemonic "COVER ABCD". In reviewing all vascular access equipment, the anaesthetist requires a systematic approach to reduce the likelihood of the problem remaining undetected, and the sub-algorithm described above is offered as a means of proceeding in this manner. This geographical approach can be commended on the strength of its being able to accommodate all the vascular access problems reported to AIMS among the first 4000 incidents. It is hoped that it will also prove to be an easily remembered and applied clinical tool, even during times of acute occupational stress for the anaesthetist.

As a complementary aide memoire in connection with incidents related to vascular access linked to drug administration problems in anaesthesia, the relevant pages developed for the Crisis Management Manual ${ }^{5}$ are displayed in the figure. These are designed to be consulted for the first time during private study time. However in the resolution of a difficult vascular access related problem, when assistance is available, they may play a role along with the other sub-algorithms in resolving an unusual, obscure, or difficult situation.

Finally, it is important that a full explanation of what happened be given to the patient, and that the problem be clearly documented in the anaesthetic record. If a particular 
precipitating event was significant, or a particular action was useful in resolving the crisis, this should be clearly explained and documented.

\section{ACKNOWLEDGEMENTS}

The authors would like to thank all the anaesthetists in Australia and New Zealand who contributed to the 4000 incident reports upon which this and the other 24 papers in the Crisis Management Series are based. The coordinators of the project also thank Liz Brown for preparing the draft of the original Crisis Management Manual Loretta Smyth for typing; Monika Bullock, RN, for earlier coding and classifying of data; Dr Charles Bradfield for the electronic version of the algorithms; Dr Klee Benveniste for literature research; Drs Klee Benveniste, Michal Kluger, John Williamson, and Andrew Paix for editing and checking manuscripts.

\section{Authors' affiliations}

R J Singleton, Senior Staff Specialist, Department of Anaesthesia and Intensive Care, Royal Adelaide Hospital and University of Adelaide, Adelaide, South Australia, Australia

S B Kinnear, Consultant Anaesthetist, private practice, Adelaide, South Australia; Visiting Anaesthetist, Flinders Medical Centre, Bedford Park, South Australia, Australia
M Currie, Clinical Quality Coordinator, Goulburn Base Hospital, Goulburn; and Consultant in Clinical Quality, Southern Area Health Service, New South Wales, Australia

S C Helps, Metabolic Neurochemistry Unit, Department of Medical Biochemistry, School of Medicine, Flinders University, Bedford Park, South Australia, Australia

This study was coordinated by the Australian Patient Safety Foundation, GPO Box 400, Adelaide, South Australia, 5001, Australia.

\section{REFERENCES}

1 Runciman WB, Webb RK, Klepper ID, et al. Crisis management: validation of an algorithm by analysis of 2000 incident reports. Anaesth Intensive Care 1993;21:579-92.

2 Webb RK, Currie M, Morgan CA, et al. The Australian Incident Monitoring Study: an analysis of 2000 incident reports. Anaesth Intensive Care 1993:21:520-8.

3 Webb RK, van der Walt JH, Runciman WB, et al. Which monitor? An analysis of 2000 incident reports. Anaesth Intensive Care 1993;21:529-42.

4 Runciman WB, Kluger MT, Morris RW, et al. Crisis management during anaesthesia: the development of an anaesthetic crisis management manual. Qual Saf Health Care 2005;14:e1.

5 Australian Patient Safety Foundation. Crisis Management Manual: COVER $A B C D$ A SWIFT CHECK. Adelaide: Australian Patient Safety Foundation, 1996, 74pp. Available at http://www.apsf.net.au/anaesthesia.htm (accessed 6 September 2004) 